\title{
A Forty Five Years Female Patient with Sclerosteosis
}

\author{
SHAMI M AHMED, ${ }^{1}$ MD. ABDUL KADER, ${ }^{1}$ QUAZI MAMTAZUDDIN AHMED, ${ }^{1}$ ABU SHAHIN, ${ }^{1}$ MD ABUL KALAM AZAD, ${ }^{2}$ \\ J AYANTA BANIK, ${ }^{3}$ SHOAYB MOMEN MOJ UMDAR, ${ }^{4}$ MOHAMMAD FERDOUS UR RAHAMAN, ${ }^{4}$ ROWSAN ARA, ${ }^{5}$ SYED \\ ATIQUL HAQ ${ }^{6}$
}

\begin{abstract}
Sclerosteosis is a rare autosomal recessive disorder, characterized by progressive, generalized bony overgrowth of the calvarium and mandible with cranial nerve entrapment and raised intracranial pressure, and abnormalities of the digits. Due to narrowing of the foramina of the cranial nerves, facial nerve palsy, hearing loss and atrophy of the optic nerves can occur. It is clinically and radiologically very similarto van Buchem disease, both of which shows generalized hyperostosis and sclerosis leading to a markedly thickened and sclerotic skull, with mandible, ribs, clavicles and also long bones. Van Buchem disease is differentiated from sclerosteosis by the absence of hand malformations and a large stature. Here we describe a case of a 45 years-old-female presented with progressive enlargement of right side of mandible, continuous pain in the right side of the face, right sided lower motor type of facial palsy, bilateral sensory neural type of hearing loss along with polydactyly and syndactyly. The final diagnosis of sclerosteosis was made by clinical features and radiological findings and exclusion of other causes of hyperosteosis. The particular interest of this paper is to present a case of sclerosteosis, and to demonstrate the differences between that entity and other sclerosing diseases of bone.
\end{abstract}

Keyword: Sclerosteosis, van Buchem disease, Hyperosteosis

\section{Introduction}

Sclerosteosis is a rare, potentially lethal, autosomal recessive skeletal disorder in which there is massive bony overgrowth leading to facial distortion, cranial nerve compression, asymmetric cutaneous syndactyly and progressive rise in intracranial pressure. ${ }^{1}$ In many cases it is associated with nail dysplasia. ${ }^{2}$ It was first recognized as a distinct entity in 1958 by Truswell, who described two unrelated South African girls with "osteopetrosis with syndactyly; a morphological variant of Albers-Schonberg disease". ${ }^{3}$ Then in 1967, Hansen first proposed the term sclerosteosis to describe the rare disorder. ${ }^{4}$ Genetic linkage analysis in two consanguineous families with sclerosteosis resulted in the assignment of the sclerosteosis gene to chromosome 17q12-q21. This chromosomal region is also responsible for another rare autosomal recessive condition with a hyperostosis similar to sclerosteosis, named van Buchem disease. ${ }^{5}$ This SOST gene is involved in the pathogenesis of two very similar conditions, sclerosteosis and van Buchem disease, by different loss of function mutations in the gene. ${ }^{6}$

\section{Case Report}

A 45-years-old muslim, married lady got herself admitted in Bangabandhu Sheikh Mujib Medical University, Dhaka, Bangladesh with the complaints of pain in the right side of the face for 7 years and progressive enlargement of right lower jaw for 1 year. She is deaf for 20 years and has right sided facial weakness for last 15 years. Pain was initially mild to moderate, later on severe for last 1 year, burning in nature, continuous, partially relieved by taking NSAIDs and radiates towards right eye, ear and right side of scalp.

On general examination she had prominent eyes. , angle of mouth was deviated to the left side and there was enlargement of lower end of right angle of mandible (Fig-1). On palpation, irregular bony swellings were revealed over right side of the mandible anterior to the angles, and below its lower border. Inner side of the mandible was thickened with marked irregularity. The upper jaw was also large and appeared to be greater in size than normal. Jaw movements were normal but crepitus was present in the right temperomandibular joint. Her left hand had six fingers and

1. Assistant Professor, Dept of Medicine, BSMMU

2. Associate Professor, Dept of Medicine, BSMMU

3. Post-graduate Trainee, Dept. of Medicine, BSMMU

4. Medical Officer, Dept. of Medicine, BSMMU

5. MD Student (Rheumatology, Thesis part), BSMMU

6. Professor \& Chairman, Dept. of Medicine, BSMMU

Correspondence: Dr. Shamim Ahmed, Assistant Professor, Dept. Of Medicine, BSMMU, E-mail: dr_shamim_ahmed@yahoo.com 
there was syndactyly of left 1st, 2nd, 3rd fingers and right 3rd, 4th fingers (Fig-2). There was swelling of soft tissue of all finger tips. She was hemodynamically stable. There was diminished sensation in all three divisions of trigeminal nerve on right side, right sided lower motor type of facial nerve palsy, bilateral sensory neural type of hearing loss. Fundoscopy was normal. Other systemic examination revealed no abnormality.

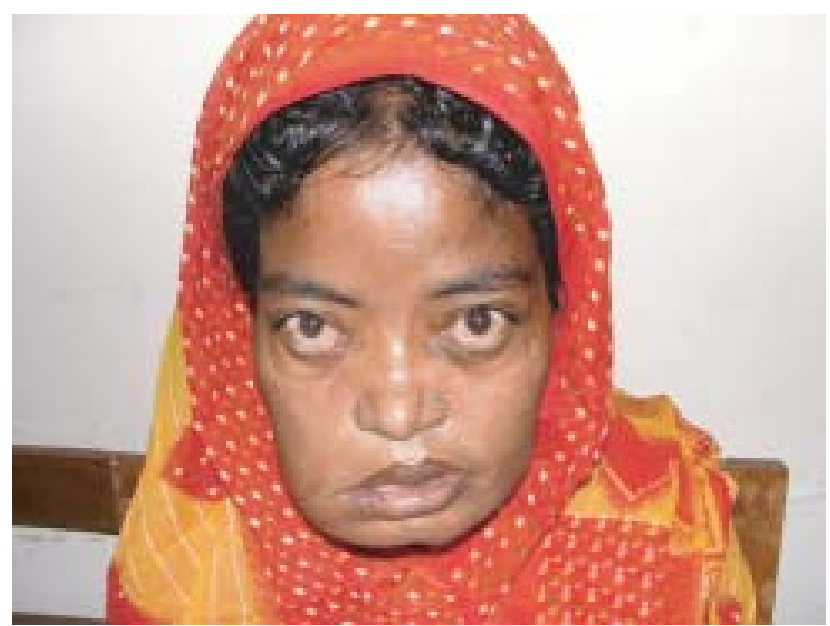

Fig.-1: Facial distorsion.

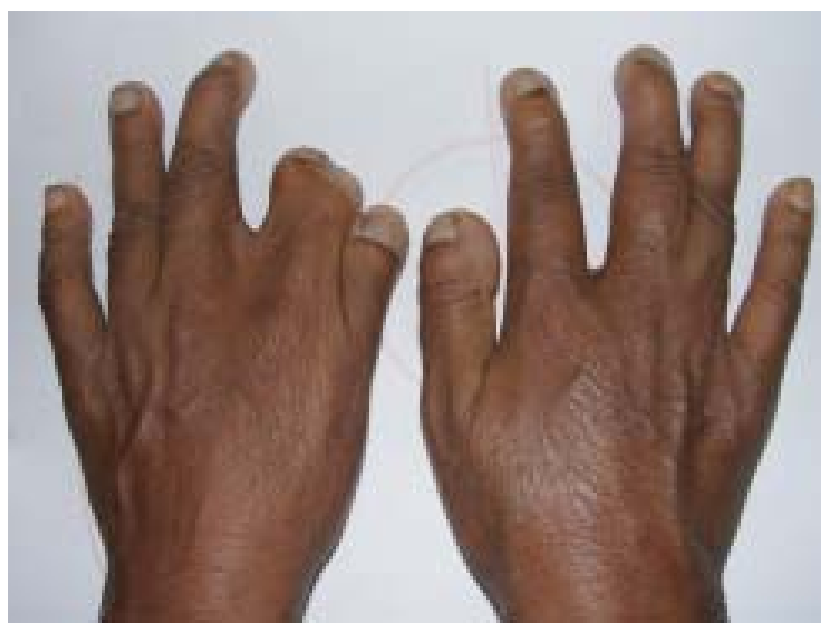

Fig.-2: Syndactyly

On investigation, complete blood count revealed- Hb\%- 11.9 g/dl, ESR- 33 mm in 1st hour, WBC- 9,300/ cumm with normal differential count. Platelet count and blood film was normal. Her FBS, SGPT, alkaline phosphatase, creatinine, and urine R/E were normal. Her TSH, S. Calcium, Phosphate was within normal limit. Basal growth hormone was 1.20 ng/ml. X-ray chest $\mathrm{P} / \mathrm{A}$ view showed increased density in the ribs and dorsal spine (Fig-3). X-ray Skull showed there was much thickening and sclerosis of the cortical bones with obliteration of diploe (Fig-4). X-ray mastoid Townes view showed symmetrical sclerosis of mandible and skull and mastoid air cells were not visible. $\mathrm{X}$ ray of long bones had increased density with endosteal cortical thickening (Fig-5). CT scan of face showed that the calvaria was thickened. The mandible and other facial bones were also thickened with increased density. The exit foramina of base of skull appeared to be narrower than usual. Soft tissue around face and scalp were normal (Fig-6).

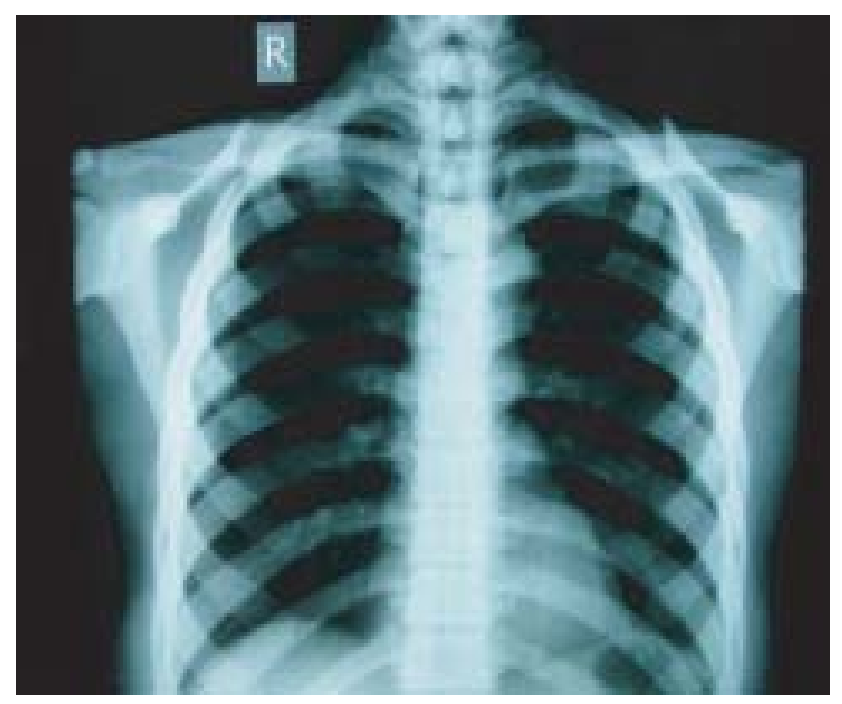

Fig.-3: $X$ ray chest showing increased density in the ribs and dorsal spine.

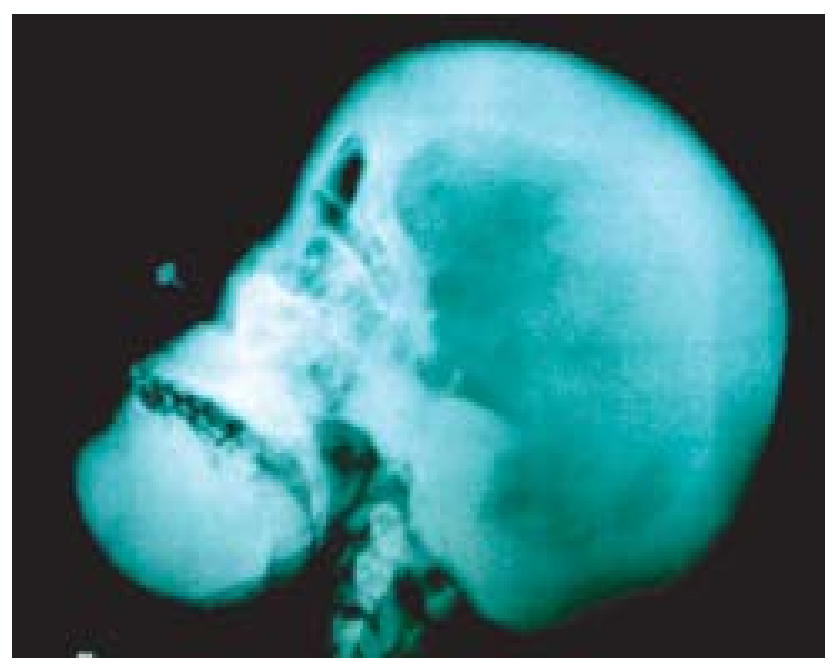

Fig.-4: $X$ ray Skull shows there is much thickening and sclerosis of the cortical bones. 


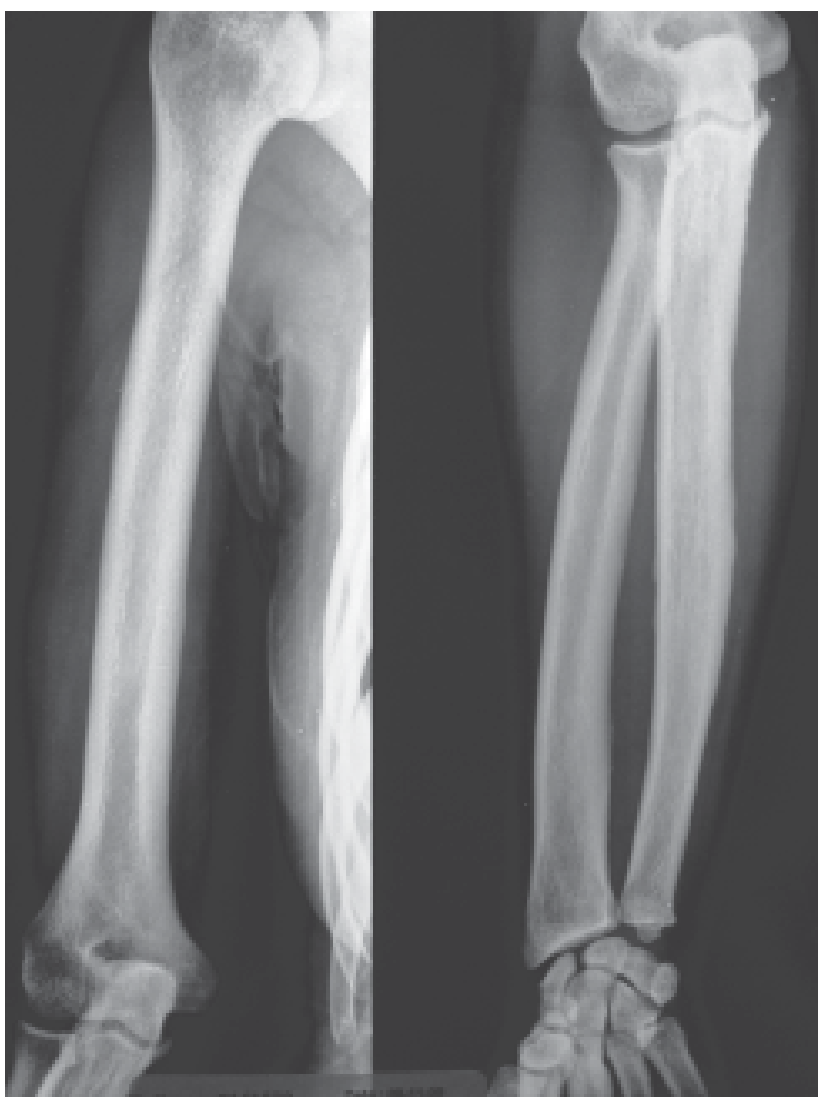

Fig.-5: $X$ ray of long bones has increased density with endosteal cortical thickening.

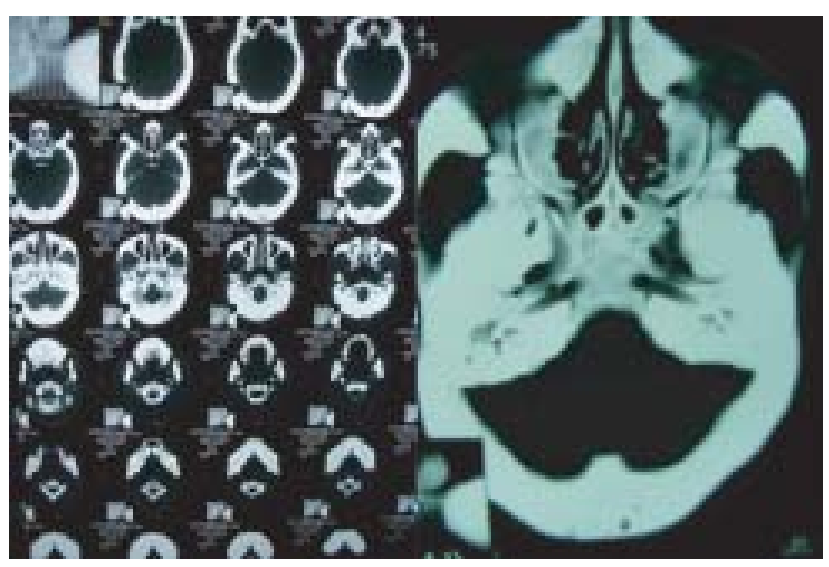

Fig.-6: CT scan of face shows the calvaria, the mandible and other facial bones are thickened with increased density.

\section{Discussion}

Bone is a dynamic tissue that is constantly remodeled by bone formation and bone resorption. Both systemic factors (such as parathyroid hormone and vitamin D) and local factors (such as transforming growth-factor $b$ and ûbroblast growth factor) maintain the balance between these processes. A diverse spectrum of pathological conditions can occur by disruption of this balance, at one end of which are the conditions that cause decreased bone density, such as osteoporosis and at the other end of this spectrum are diseases that cause increased bone mass or density. The disorders causing high bone mass are called "osteoscleroses" if trabecular bone is increased and "hyperostoses" if cortical bone is increased. The latter include conditions such as osteopetrosis (Albers Schonberg disease), progressive diaphyseal dysplasia (CamuratiEngelman disease), hyperostosis corticalis generalisata (Van Buchem's disease), sclerosteosis, as well as many others. ${ }^{7}$

In the group with increased bone density, sclerosteosis is a rare autosomal recessive skeletal dysplasia and the majority of the cases have been described in the Afrikaner community of South Africa. The determinant gene (SOST) responsible for this condition skeletal dysplasia and the majority of the cases have been described in the Afrikaner community of South Africa. The determinant gene (SOST) responsible for this condition has been isolated. The anomalies of affected persons are progressive and may be present in early childhood. ${ }^{8}$ Documentation of the natural history of the condition in 63 affected individuals in South Africa, over a 38-year period, shows that it is a severe disorder which places considerable burden upon affected individuals and their families. ${ }^{9}$ Molecular studies showed a very restricted expression pattern of SOST and its gene product, sclerostin, the physiological role of which is most likely the suppression of bone formation. Therefore, the identification of sclerostin as an important protein in bone metabolism might become an important tool in the development of therapeutic strategies to prevent and treat osteoporosis. ${ }^{10}$

The clinical features of this condition can be observed in infancy. The typical facies is characterized by a steep, high forehead, ocular hypertelorism, broad flat root of the nose, and prognathic, broadened, squared mandible with normal body proportions. ${ }^{10}$ These deformities progress with the age and affected children are tall for their age and adults may have gigantism. Commonly, there is bilateral but often asymmetric cutaneous, partial or total syndactyly, usually of the second and third fingers, with deviation of the terminal phalanges. The nails of the involved fingers and toes are often dysplastic or absent. ${ }^{3}$ Another important clinical feature is involvement of cranial nerves by progressive bony encroachment. Almost all cases have transient facial-nerve paralysis and characteristically it is unilateral for many years. Bony encroachment in the middle ear cavities and auditory nerve canals often causes deafness and compression of the 
optic nerves can cause optic atrophy, reduction in visual field, convergent strabismus, and nystagmus in some cases. Exophthalmos may also be present. ${ }^{4}$ Progressive diminution of the capacity of the cranial cavity with rise of intracranial pressure occurs by bony overgrowth of the calvarium and several patients have died suddenly from impaction of the medulla oblongata in the foramen magnum. Fractures are infrequent and bones are resistant to trauma. ${ }^{3}$

The radiographic findings of sclerosteosis are pathognomonic although the clinical picture suggests the diagnosis. The most notable changes occur in the skull which shows the calvarium is widened and uniformly sclerotic, the base is very dense and the cranial nerve foramina are narrowed and the sella turcica may be expanded. The mandible is also diffusely sclerotic and grossly thickened with asymmetrical distortion and dental malocclusion. ${ }^{3}$ The clavicles and ribs are widened and dens, the scapulae, pelvis, vertebral bodies, and long bones are uniformly sclerotic with cortical hyperostosis and without any alteration of shape. ${ }^{4}$ Haematology and urinalysis are usually normal. Serum calcium and phosphorus are usually normal but the alkaline phosphatase level may be slightly raised. In this disorder pituitary function (growth hormone, adrenocorticotrophin, gonadotrophin and thyrotrophin) and calcium 'homeostasis' are normal. ${ }^{11}$ Bone mineral density (BMD) is markedly increased at all skeletal sites in Individuals with sclerosteosis. ${ }^{12}$

Our patient presented to us with the features of enlarged mandible, right sided $7^{\text {th }}$ nerve, pulsy, bilateral $8^{\text {th }}$ nerve pulsy and syndactyly of left thumb, index and middle fingers and right index and middle fingers. The radiographic findings are typical of sclerosteosis. The considered differential diagnosis are Paget's disease of bone, osteopetrosis, progressive diaphyseal dysplasia (Camurati-Engelman disease), hyperostosis corticalis generalisata (Van Buchem's disease), Osteopathia striata with generalized hyperostosis, Osteopathia striata with skull thickening, Myelosclerosis, Halliday's hyperostosis.

Paget's disease of bone can be easily excluded by the appearance of overall density of the skull in X-ray. In Paget's disease it is usually of cotton-wool appearance associated with changes in other bones of the skeleton obscuring the basic anatomy of the bone along with an elevated serum alkaline phosphatase level. In Osteopetrosis the radiographic finding of uniformly dense and sclerotic bone is usually found in the epiphyses, metaphyses and diaphyses of long bones which are extremely brittle and liable to fracture easily. ${ }^{13}$ In our patient there is no fracture despite the long history.

In Camurati-Engelman disease, the parietal and petrous temporal bones, the facial bones and jaws are not involved and a characteristic deposition of excess subperiosteal bone occurs in the mid-shaft of the long bones with normal ends. The full picture of the disease shows bony alterations, muscle weakness and neurological signs. ${ }^{13}$ In this case, despite the advanced radiological changes there were no signs of muscular weakness. Van Buchem disease (hyperostosis corticalis generalisata) is only excluded due to the presence of syndactyly. Another differential diagnosis may be acromegaly because of clinical resemblance of enlarged mandible but radiological changes exclude it.

The diagnosis of Osteopathia striata with generalized hyperostosis is made from the presence of a coarse striation in the long bones and pelvis and when it is associated with gross hyperostosis of the skull it is called Osteopathia striata with skull thickening. ${ }^{14}$ In our case $\mathrm{X}$ ray of long bones do not show any striation. In Myelosclerosis patchy sclerosis is seen but the skull is not affected and haematological changes are present and in Halliday's hyperostosis the mandible is less affected and the long bones are not hyperostotic. ${ }^{14}$ So both these conditions are excluded.

There is no curative therapy for the disease. In early adulthood prophylactic craniectomy may be done to remove raised ICP. Surgical treatment for both decompression of seventh and eighth cranial nerves and recontouring of the mandible has been performed but the results are inconsistent. An external hearing aid may be helpful. Syndactyly can be removed by cosmetic repair and for dental malalignment orthodontic measures are indicated. ${ }^{3}$

\section{Conclusion}

Sclerosteosis is a rare autosomal recessive, progressively leathal skeletal dysplasia which occurs due to loss of function mutation of the SOST gene in chromosome 17q12q21. Clinically and radiologically it is very similar to Van Buchem disease which also occurs due to loss of function mutation of the same gene and can only be separated clinically by the presence of syndactyly and gigantism, which is present in sclerosteosis. The facial appearance of the disease resembles acromegaly and paget's disease because of jaw changes but knowing the condition might help the clinicians that sclerosteosis is another possibility.

\section{Conflict of interest: None}

\section{References}

1. Beighton P, Davidson J, Durr L, Hamersma H; Sclerosteosis - an autosomal recessive disorder. Clin Genet 1977; 11(1): $1-7$.

2. Itin PH, Keserü B, Hauser V. Syndactyly/brachyphalangy and nail dysplasias as marker lesions for sclerosteosis. Dermatol 2001; 202(3): 259-60. 
3. Beighton P. Sclerosteosis. J Med Genet 1988; 25: 200-03.

4. Sugiura Y, Yasuhara T. Sclerosteosis. A case report. J Bone Joint Surg Am. 1975; 57: 273-77.

5. Balemans W, Van Den EJ, Freire Paes-Alves A, et al. Localization of the gene for sclerosteosis to the van Buchem disease-gene region on chromosome 17q12-q21; Am J Hum Genet 1999; 64(6): 1661-69.

6. Balemans W, Ebeling M, Patel N, et al. Increased bone density in sclerosteosis is due to the deficiency of a novel secreted protein (SOST); Human Molecul Genetics 2001, 10(5); 537-43.

7. Van Hul W, Balemans W, Van Hul E, et al. Van Buchem Disease (Hyperostosis Corticalis Generalisata) Maps to Chromosome 17q12-q21; Am J Hum Genet1998; 62: 391-99.

8. Bueno M, Oliván G, Jiménez A, et al.; Sclerosteosis in a Spanish male: first report in a person of Mediterranean origin; J Med Genet 1994; 31: 976-77.
9. Hamersma H, Gardner J, Beighton P. The natural history of sclerosteosis. Clin Genet. 2003; 63(3): 192-97.

10. Balemans W, Van Hul W; Identification of the disease-causing gene in sclerosteosis-discovery of a novel bone anabolic target? J Musculoskelet Neuronal Interact. 2004; 4(2):13942.

11. Epstein S, Hamersma H, Beighton P. Endocrine function in sclerosteosis; S Afr Med J 1979; 55(27): 1105-10.

12. Gardner JC, van Bezooijen RL, Mervis B, et al. Bone mineral density in sclerosteosis; affected individuals and gene carriers; J Clin Endocrinol Metab. 2005; 90(12): 6392-5.

13. Dyson DP. Van Buchem disease (hyperostosis corticalis generalisata familiaris); A case report; British J Oral Surg I972; 9: 237-45.

14. Jacobs P. Van Buchem disease; Postgrad Med J 1977; 53: 497-506. 